\title{
The Application of Integral Source Model in The Design of Freeform Optics for Several Multidirectional Light Sources"
}

\author{
Nikolay Bogdanov [0000-0003-1221-8827], Igor Potemin [0000-0002-5785-7465], \\ Dmitry Zhdanov [0000-0001-7346-8155], Yan Wang [0000-0002-3026-2306] \\ ITMO University, 49 Kronverksky Pr., St. Petersburg, 197101, Russia \\ nnbogdanov@itmo.ru, ipotemin@yandex.ru, ddzhdanov@mail.ru, \\ awoptics@yandex.ru
}

\begin{abstract}
One of the features of an intelligent transport system is the formation of communication channels between vehicles. Vehicle-to-vehicle communication will help reduce the number of road accidents. Li-Fi technology is considered as a method for communication. Li-Fi uses visible light for data transmission. A single source of radiation may not be sufficient to provide a certain signal level at the receiver, so multiple sources must be used. Also, signal transmission should be in all directions in the horizontal plane. The study addresses the problem of designing optical systems of circular radiation with several multidirectional sources. It proposes the modification of the ray mapping method for the task of designing optical elements for the Li-Fi wireless communication technology between vehicles. Also, it describes the algorithm for calculating optical systems of circular radiation for a signal source and signal receiver. Finally, the results of calculating and virtual prototyping of devices designed by the proposed method.
\end{abstract}

Keywords: Nonimaging Optics, Freeform Surfaces, Optical Wireless Communication, Li-Fi.

\section{Introduction}

Currently, there is an increase in the number of vehicles on the roads, which causes certain traffic control problems. In the year of 2014, the global vehicle fleet consisted of more than 1.25 billion vehicles [1]. The number of vehicles on the road as well as the number of accidents is growing every day. According to the World Health Organization (WHO), more than 1.2 million people are killed and another 20-50 million are

Copyright (C) 2020 for this paper by its authors. Use permitted under Creative Commons License Attribution 4.0 International (CC BY 4.0).

This work was partially supported by Russian Foundation for Basic Research (project No. 1808-01484). 


\section{N. Bogdanov, I. Potemin et.al}

injured in road vehicle accidents every year [2]. To prevent accidents and reduce traffic, it is essential to make the transport system intelligent. One of the features of the intelligent transport system (ITS) is a vehicle to vehicle communication (V2V). V2V communication technology allows vehicles to broadcast and receive signals in all directions (up to 10 times per second), creating 360-degree «awareness» of other vehicles [3, 4]. Possible transmitted messages include the following data:

1. Vehicle speed.

2. Car location and the direction of its movement.

3. Driving lane change.

4. Information about stability control and traction control.

5. Information about the braking system and antiskid braking system.

An equally important scope of development in terms of ITS is autonomous vehicles, or in other words, self-driving cars [5]. In order for a vehicle to become autonomous, it is necessary to assess the situation on the road while driving and track other vehicles nearby. One needs to exchange data with nearby cars for this to occur. Thus, the vehicle communication is an important part of ensuring the autonomy of the vehicles.

At the moment, there are numerous research papers on various types of such communication between vehicles based on DSRC, Wi-Fi [6-9] and Li-Fi technology [10, $11,12]$.

$\mathrm{Li}$-Fi uses visible or invisible optical wireless communication links that provide high-speed data transmission with low latency over spatially defined communication links, which makes it possible to design cellular networks and reduce cross-channel interference. Various aspects of the Li-Fi technology have developed rapidly over the past decade: various modulation schemes have been investigated [13], new emitters have been developed [14], and the integration of Li-Fi into existing networks has been studied [15]. To provide the vehicle communication via $\mathrm{Li}-\mathrm{Fi}$, a number of works suggest using lighting equipment on the front and back of vehicles, as well as integrating it into traffic lights [16-20]. In these studies, the main focus is on electrical circuits and on common circuits for constructing communication networks.

However, despite the active development of $\mathrm{Li}$-Fi and the attempts to implement this technology for wireless vehicle communication, the present-day methodology of optical design for Li-Fi has been barely considered. As a rule, simple spherical lenses can be used for both a radiation source and a receiver. However, they may not fully correspond to current trends, such as the miniaturization of $\mathrm{Li}-\mathrm{Fi}$ transceivers to allow their integration into other devices. Scaling down the optics directly affects performance. The use of freeform optics can compensate for performance degeneration when developing compact structures customized for a specific application.

A few works are devoted to the methods of designing refractive optical systems with freeform surfaces for radiation sources as well as radiation receivers within the $\mathrm{Li}$-Fi communication systems. Thus, [21, 22] demonstrate the potential of modern optics with freeform surfaces for Li-Fi technology.

Methods of designing optics for the radiation source and radiation receiver are discussed. An example of calculated optical systems confirms the effectiveness of the ray 
The Application of Integral Source Model in The Design of Freeform Optics... 3

mapping method [23, 24]. In [25], a hybrid optical system is proposed for a nearby source and receiver of radiation.

The authors estimate the signal loss when the receiver is displaced from the source by a certain distance for the proposed optical system and compare it with a design in which the source and receiver are separated and each corresponds to its own optical system with spherical surfaces. The proposed optical systems have some disadvantages, such as:

1. The narrow range of observation of the receiver' optical system is a radiation halfangle of $17^{\circ}$. Systems operate at a small shift from the receiver optical axis from the source.

2. The use of a single combined system does not provide signal transmission and reception in all directions in the horizontal plane, which is necessary to ensure communication between vehicles.

3. For $360^{\circ}$ radiation in the horizontal plane, one should use several optical systems with radiation sources.

4. Optical systems have not been tested for efficiency at long distances, which may correspond to the distances between vehicles during vehicle communication using the Li-Fi technology.

It is also worth noting that the experimental application of the Li-Fi technology with help of the vehicle front and rear lights does not provide signal transmission and reception in all directions in the horizontal plane, which is a significant limitation in communication between nearby vehicles.

This paper proposes a method for designing hybrid optical systems that emit and receive radiation from all directions in the horizontal plane, and also provides an example of using such systems for the communication between vehicles using the Li-Fi technology.

\section{The initial optical scheme}

As mentioned earlier, the signal should be transmitted in all directions in the horizontal plane; simultaneously, the photodetector should be able to receive the signal from any direction in the horizontal plane. The use of a single radiation source imposes restrictions on the transmitted signal power; for this purpose, several sources have to be used to form a more powerful signal. The proposed layout of sources and their orientation is shown in Figure 1.

LEDs are considered as radiation sources. The centers of the sources are located along a circle of a certain radius, the radiation direction of each source is oriented at a certain angle relative to the radiation direction of other sources so that the signal is transmitted in a horizontal plane at $360^{\circ}$.

In such a system, both horizontal and vertical disposition of the photodetectors is possible, that make it possible to receive a signal from all directions in the horizontal plane. In this paper, we will consider the horizontal disposition of the photodetector. 
4 N. Bogdanov, I. Potemin et.al

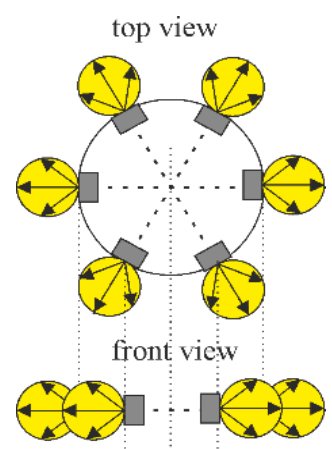

Fig. 1. Spatial disposition of radiation sources

The designed optical system is proposed to be placed on a vehicle roof to make the transmission and reception of a signal at $360^{\circ}$ in the horizontal plane possible. Based on the size of passenger cars and the range of distances at which the Li-Fi system should operate, the field of view for the signal source and receiver can be determined. The height range of passenger cars is 1,300-2,100 mm. In this study, the minimum distance between vehicles is taken as $3 \mathrm{~m}$ and the maximum distance - as $10 \mathrm{~m}$. These distances are determined based on the recommendations of experts and traffic regulations, which make it possible to avoid a collision with another vehicle. Graphically, the system operating range can be represented by the scheme shown in Fig. 2. It follows that one can determine the required maximum radiation angle at which the radiation will propagate in the specified range. In this case, the half angle of radiation is $15^{\circ}$.

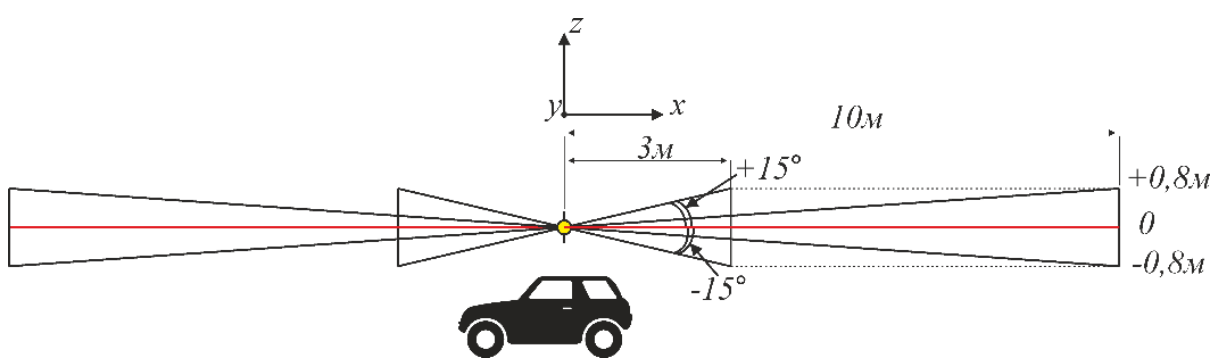

Fig. 2. Li-Fi system operating range

The initial design of optical systems for an emitter and a receiver is shown in Fig. 3a and Fig. 3b. The proposed scheme of the designed optical element for the emitter includes two working surfaces: the first one is cylindrical, and the second one is a freeform surface, which will be calculated. Light sources are located along a circle of a certain radius and are oriented at a certain angle. There can from 6 and more sources of light; the maximum number is determined based on the overall dimensions of the sources and restrictions on the overall dimensions of the optical system. The proposed scheme of the optical element for the receiver includes two freeform surfaces. The photodetector is placed in the center of the optical system. 


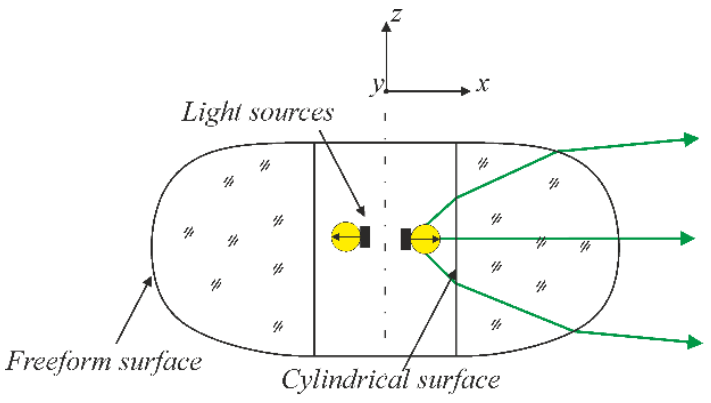

a

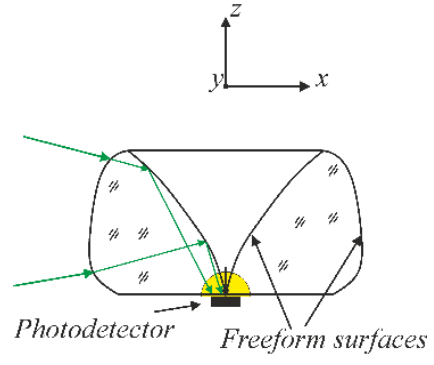

b

Fig. 3. Optical schemes for the Li-Fi emitter (a) and for the Li-Fi receiver

\section{Modified ray mapping method. Integral model of radiation source}

The classical ray mapping method works effectively for a single point of Lambertian radiation source. It is necessary to clarify the features of the coordinate system and calculation the total luminous flux for such a case. The light intensity of such sources is proportional to the cosine of the angle between the normal to the radiating surface and the direction of radiation. The value of the radiation flux in the range of angles $\varphi_{1}$ $-\varphi_{2}, \alpha_{1}-\alpha_{2}$ emitted by such a source is calculated by the formula [26]:

$$
\Phi=\int_{\varphi_{1}}^{\varphi_{2}} d \varphi \int_{\alpha 1}^{\alpha 2} I_{0} \cdot \cos \alpha \cdot \sin \alpha d \alpha
$$

In the case of full light flux, the azimuthal angle $\varphi$ will change in the range $[0 ; 2 \pi]$, the polar angle $\alpha$ will change in the range $[0 ; \pi / 2]$, and counted from the vertical plane, I0 is the light intensity in the direction $(\alpha=0)$ perpendicular to the emitting surface of the LED. The coordinate system used in expression (1) is shown in Figure 4, when the radiating surface of the source is parallel to the horizontal plane, and the polar axis is pointed to the zenith. The way it is disposed, the maximum value of the light intensity I0 will correspond to the vertical direction.

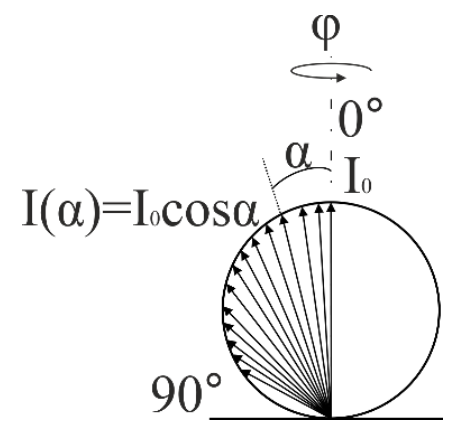

Fig. 4. Light distribution curve for Lambertian radiation source 


\section{N. Bogdanov, I. Potemin et.al}

However, the classical ray mapping method cannot be applied directly for the case when several multidirectional radiation sources are used in the system. To calculate an optical system with a single free-form surface, the ray mapping method must be modified. The algorithm for designing an optical system with free-form surfaces for several multidirectional radiation sources is shown in Figure 5.

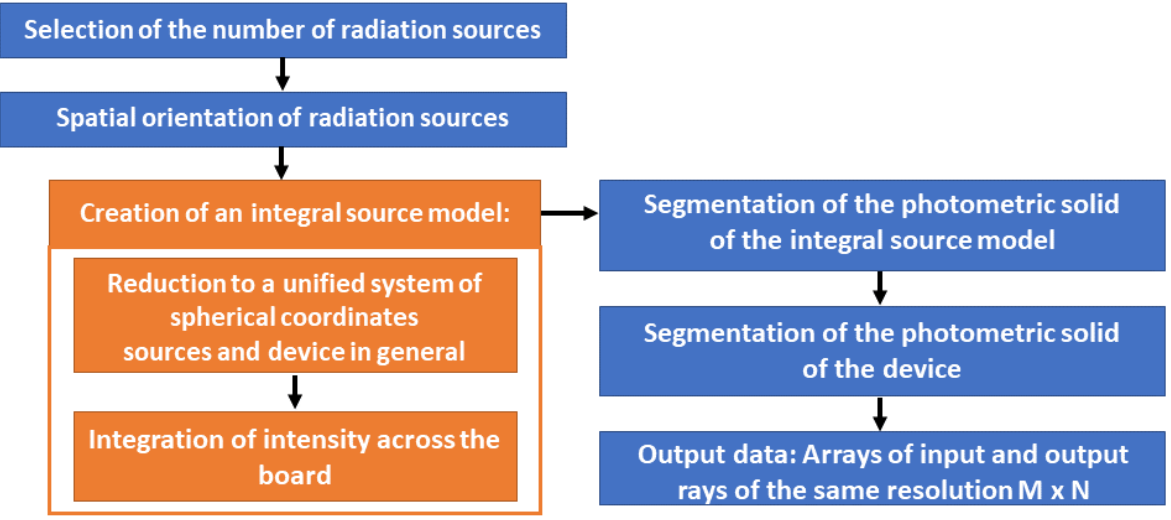

Fig. 5. Algorithm of the modified ray mapping method

At first, it is determined how many sources will be used and how they will be oriented in space. The quantity of radiation sources is determined based on the data on the optical power of one source and the required optical power from the device. The use of several sources allows to increase the optical power of the emitted signal and transmit over longer distances than could be transmitted by only one source.

The next step is the main feature of the proposed method - creation of an integral source model. Radiation sources are considered not separately, but as a single integral source model, for which the radiation intensities in all directions are known. At this stage, it is necessary to consider in more detail the features and process of forming the integral source model.

The integral source model is a table function in which the radiation intensity is determined for all specified directions. The orientation of the coordinate system in the integral source model and in the device must be the same since the correctness of the calculations at further steps depends on this. Coordinate system orientations are shown in the figure 6. According to the proposed design of the optical system and the disposition of several radiation sources, the maximum light intensity should correspond to the directions lying in close proximity to the horizontal plane, as shown in Figure 3a (arrows indicate the direction corresponding to the maximum light intensity $\mathrm{I}_{0}$ ). But with this orientation, the rotation axis of the light distribution curve (LDC) of the light source at the angle $\varphi$ is perpendicular to the rotation axis of the LDC at the angle $\varphi^{\prime}$ of the LiFi module transmitter device as a whole (see Figure 6a). The coordinates of each radiation source are modified so that the direction of the source rotation axis coincides with the rotation axis of the LDC output radiation, and the elevation angles $\theta$ are counted from the horizontal plane, where the radiation direction in the horizontal plane will 
correspond to the angle $\theta=0^{\circ}$, in the direction of the zenith $\theta=+90^{\circ}$, in the direction of the nadir $\theta=-90^{\circ}$ (see Figure $6 \mathrm{~b}$ ).

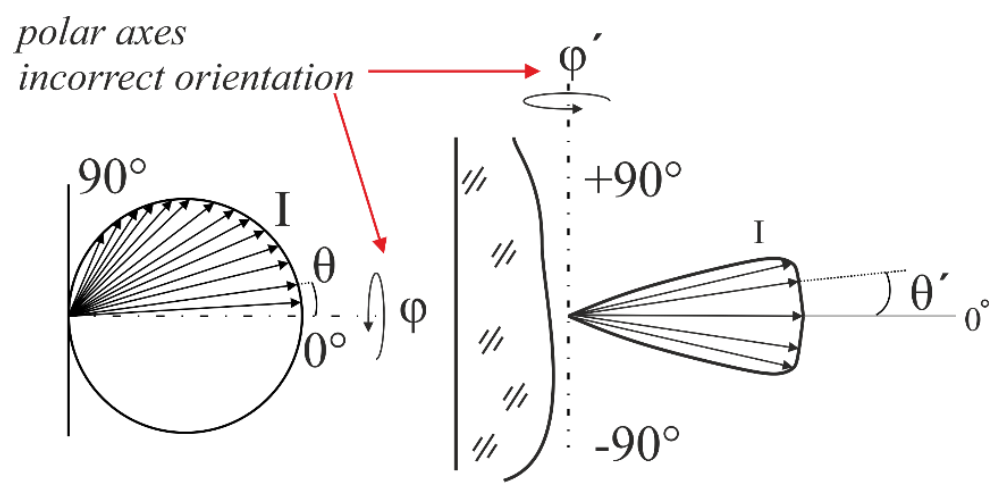

a

polar axes correct orientation
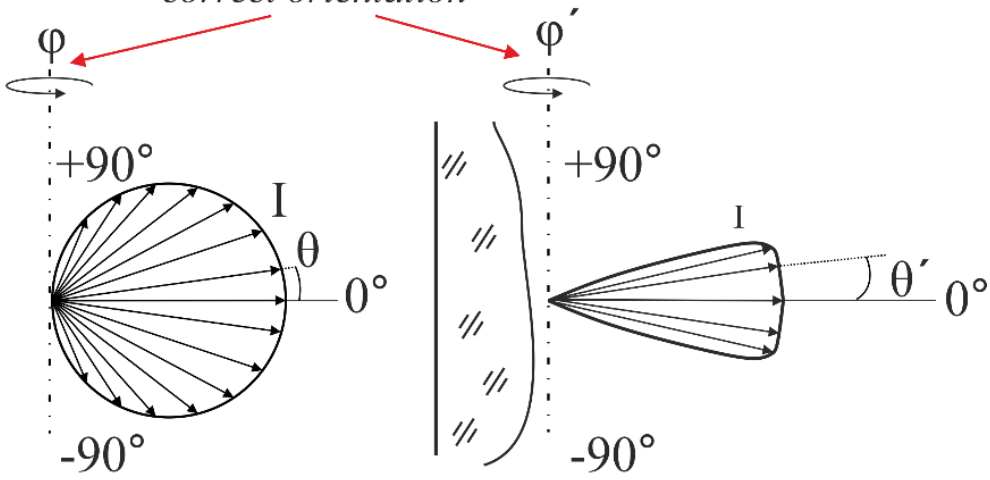

$\mathrm{b}$

Fig. 6. Coordinate systems orientations for radiation source(left) and device (right)

As a result of modifying the source coordinate system, the formula (1) for calculating the radiation flux in a given range of angles will take the following form:

$$
\Phi=\int_{\varphi_{1}}^{\varphi_{2}} d \varphi \int_{\theta 1}^{\theta 2} I_{0} \cdot \cos \theta \cdot \cos \varphi \cdot \cos \theta d \theta
$$

In the case of full radiation flux, the azimuthal angle $\varphi$ changes in the range $[-\pi / 2$; $+\pi / 2]$, and the elevation angle $\theta$ changes in the range $[-\pi / 2 ;+\pi / 2]$.

At the next sub step, the radiation intensities are summarized in all directions. The diagram (Fig. 7) shows the angle range $\varphi^{\prime}$ for the optical system of circular radiation and the angle ranges $\varphi$ for each radiation source. It can be seen from the diagram the radiation from which sources coincides in the direction for each angle $\varphi^{\prime}$ of the circular radiation system. If the radiation directions coincide, the intensity from each source will be summed up. For example, in the range of angles $\varphi^{\prime}$ from $0^{\circ}$ to $30^{\circ}$, the total light 


\section{N. Bogdanov, I. Potemin et.al}

intensity of the circular radiation system will be comprised of the light intensities of sources $1,2,6$, since each of these sources emits light in the directions of the range of angles $\varphi^{\prime}$. The same will happen for all the ranges. The result is a table function I $(\theta, \varphi)$ for the system, and the full radiation flux will take the following form:

$$
\Phi=\int_{\varphi_{1}}^{\varphi_{2}} d \varphi \int_{\theta 1}^{\theta 2} I(\theta, \varphi) \cos \theta d \theta
$$

$\varphi^{\prime}$ scale for circular radiation system

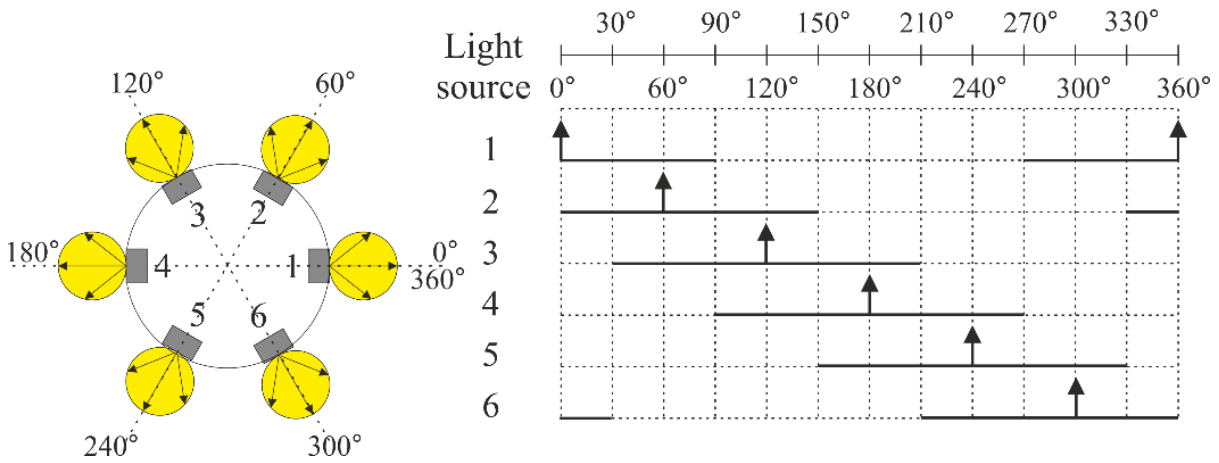

Fig. 7. The radiation diagram for 6 sources and their radiation crossing

The photometric solid of the circular radiation system device is defined by a table function. The radiation intensity is determined for all directions $\theta^{\prime}$ and $\varphi^{\prime}$. The radiation flux is calculated by the formula (3), where I $\left(\theta^{\prime}, \varphi^{\prime}\right)$ is a table function, the azimuthal angle $\varphi$ ' changes in the range $[0 ; 2 \pi]$, the elevation angle $\theta$ ' changes in the range $[-\pi / 6 ;+\pi$ /6], as it was established from Fig. 2.

After determining integral source model photometric solid and required photometric solid, it is necessary to segment them, that is, to divide it into several equal radiation fluxes. This operation is required to determine the corresponding boundaries for the angles $\varphi, \theta$, in the direction of which the light flux is emitted. The operation of photometric solid segmentation for both is the same, so we describe the procedure once.

For numerical calculation of the integral, the boundary values of the angle ranges $\varphi$, $\theta$ are pre-assigned and the subintegral table function $\mathrm{I}(\theta, \varphi)$ is defined. Next, it should be determined how many segments the photometric solid should be divided into. If by $\theta$ the number of segments is $N$, and by $\varphi-M$, the values of elementary flows will be as follows:

$$
\begin{gathered}
\Phi_{n}=\frac{\Phi}{N} \\
\Phi_{m}=\frac{\Phi}{M}
\end{gathered}
$$

To find the corresponding angles in which the flows of $\Phi_{\mathrm{n}}, \Phi_{\mathrm{m}}$ are enclosed, it is necessary to perform numerical integration of two integrals, having previously assigned step $\mathrm{d} \varphi, \mathrm{d} \theta$. 
The Application of Integral Source Model in The Design of Freeform Optics... 9

$$
\begin{aligned}
\Phi_{n}^{\prime} & =\int_{\varphi_{1}}^{\varphi_{2}} d \varphi \int_{\theta i}^{\theta i+1} I(\theta, \varphi) \cos \theta d \theta \\
\Phi_{m}^{\prime} & =\int_{\varphi_{i}}^{\varphi_{i+1}} d \varphi \int_{\theta 1}^{\theta 2} I(\theta, \varphi) \cos \theta d \theta
\end{aligned}
$$

The value of $\Phi^{\prime}{ }_{n}$ is compared with $\Phi_{n}$, if it is equal to or close to the desired value, the current angle $\theta_{i+1}$ is written; otherwise, it is increased by the value $\mathrm{d} \theta$ and the integral is calculated again.

After the segmentation of both the photometric solid of the light source and the specified photometric solid is completed, arrays of input and output rays of the same resolution $\mathrm{M} \mathrm{x} \mathrm{N}$ are formed. Each array element has a row number and a column number (i, j). Array elements are defined as light rays with specified orientation in space (angles $\theta, \varphi)$. Each (i, j)-th ray of the light source has a corresponding output $(i, j)$-th ray. Each incoming ray with index $(i, j)$ and direction $(\theta, \varphi)$ corresponds to an output ray with index $(i, j)$ and direction $\left(\theta^{\prime}, \varphi^{\prime}\right)$. To construct a surface, one should perform a series of calculations for each element of the ray array, and perform this process sequentially from element to element. An array of rays can be traversed by rows or columns, for example, for each $\varphi$, one can calculate the coordinates of the surface vertices for all angles $\theta$ in the range [ $\theta \min , \theta \max ]$.

\section{Constructing a freeform surface}

Let us consider the optical path through two refractive surfaces, which is shown in Figure 8.

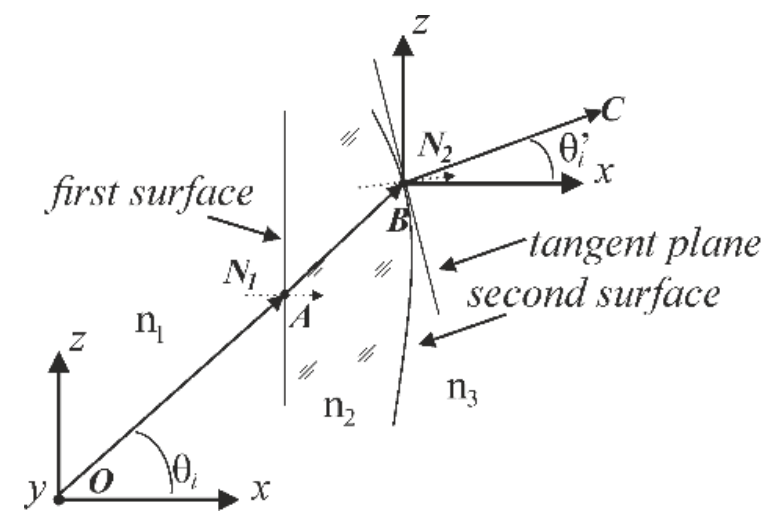

Fig. 8. the optical path through two refractive surfaces

The first surface refracts the incident ray OA (with the angle of incidence $\theta_{\mathrm{i}}$ ) into the ray $A B$. The second surface refracts the ray $A B$ into the beam $B C\left(\theta_{i}{ }^{\prime}\right)$. The refraction on the first surface is determined by Snell's law. To determine the coordinates of the vertex of the second surface, it is necessary to solve a set of equations on the basis of Figure 9. 
10 N. Bogdanov, I. Potemin et.al

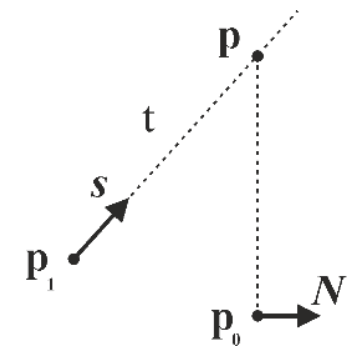

Fig. 9. beam-plane intersection scheme

Point $\boldsymbol{p}_{\boldsymbol{1}}$ belongs to the first surface, point $\mathbf{p}_{\mathbf{0}}$ belongs to the second surface, $\mathrm{N}$ is the normal at point $\mathbf{p}_{0}, \mathbf{s}$ is the unit vector, $t$ is the scalar that defines the distance to point $\mathbf{p}$, point $\mathbf{p}$ is unknown and belongs to the second surface.

$$
\begin{gathered}
\left\{\begin{array}{c}
\left(\boldsymbol{p}-\boldsymbol{p}_{\mathbf{0}}\right) \times \boldsymbol{N}=0 \\
\boldsymbol{p}=\boldsymbol{p}_{1}+\boldsymbol{s} \cdot t
\end{array}\right. \\
\boldsymbol{p}_{1} \times \boldsymbol{N}+\boldsymbol{s} \cdot t \times \boldsymbol{N}-p_{0} \times \boldsymbol{N}=0 \\
t=\frac{\left(\boldsymbol{p}_{0}-\boldsymbol{p}_{1}\right) \times \boldsymbol{N}}{\boldsymbol{s} \times \boldsymbol{N}} \\
\boldsymbol{p}=\boldsymbol{p}_{\mathbf{1}}+\boldsymbol{s} \cdot t
\end{gathered}
$$

As is obvious, the normal $\boldsymbol{N}$ belongs to the previous point $\mathbf{p}_{0}$ and the very first point po should be assigned. In Figure 10, one can see points $\boldsymbol{A}_{\boldsymbol{0}}$ and $\boldsymbol{B}_{\boldsymbol{0}}$, which, according to the previous diagram, are points $\mathbf{p}_{\mathbf{1}}$ and $\mathbf{p}_{\mathbf{0}}$. Point $\boldsymbol{A}_{\boldsymbol{0}}$ is set by crossing the extreme ray and the surface, point $\boldsymbol{B}_{\boldsymbol{0}}$ is assigned arbitrarily, but in such a way that the thickness of the optical element is not zero and lies along the course of the refracted ray. The orientation of the $\boldsymbol{N}_{20}$ normal at $\boldsymbol{B}_{\boldsymbol{0}}$ is also assigned arbitrarily, but this will affect the thickness of the optical element. This is how all the points for a single section are found, then the coordinates of the vertices are calculated for each $\varphi$.

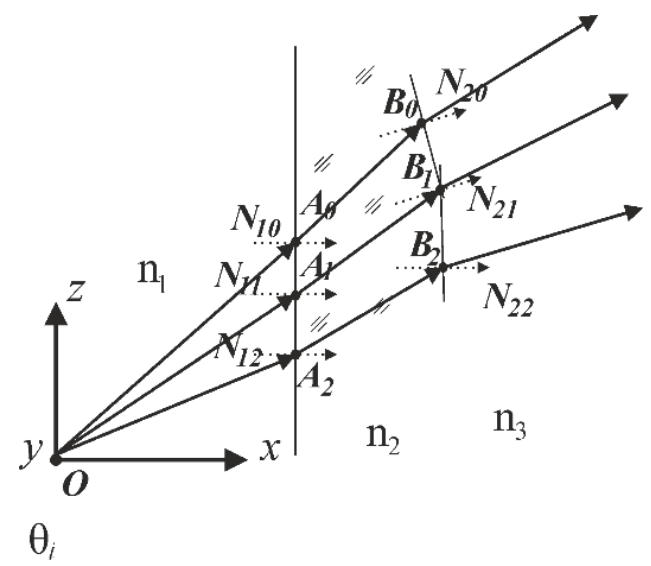

Fig. 10. scheme for finding surface coordinates for several rays 
The Application of Integral Source Model in The Design of Freeform Optics... 11

\section{$5 \quad$ Method implementation}

In accordance with the elaborated method, a program with the help of which the optical system for the source was calculated was implemented in the Python programming language. To calculate the optical system of the receiver, we used the method previously described in [27], according to which the freeform surface profile is calculated, and then a three-dimensional model is formed by rotation.

Optical modeling was performed using a hybrid software complex Lumicept [28]. During the simulation, Fresnel reflections on optical surfaces were taken into account. Infrared LEDs Osram SFH 4170S A01 [29] with the optical power of 1,400 mW each are used as emitters. The receiver includes a Hamamatsu S10784 [30] photodiode with a spherical lens.

The elaborated optical systems are shown in Fig. 11. The model used 12 extended light sources with a Lambert diagram of the light intensity distribution. The diagrams are oriented in such a way that the angle between the directions with the maximum light intensity of nearby sources was $60^{\circ}$. It emits 2 sources in each direction. The optical power of each source is $1,400 \mathrm{~mW}$. The number of sources was selected according to the condition of ensuring the minimum-signal level on the photodiode at the maximum distance. The calculated optical systems are polygonal objects in the «obj» format, which is widely used in CAD systems [31].
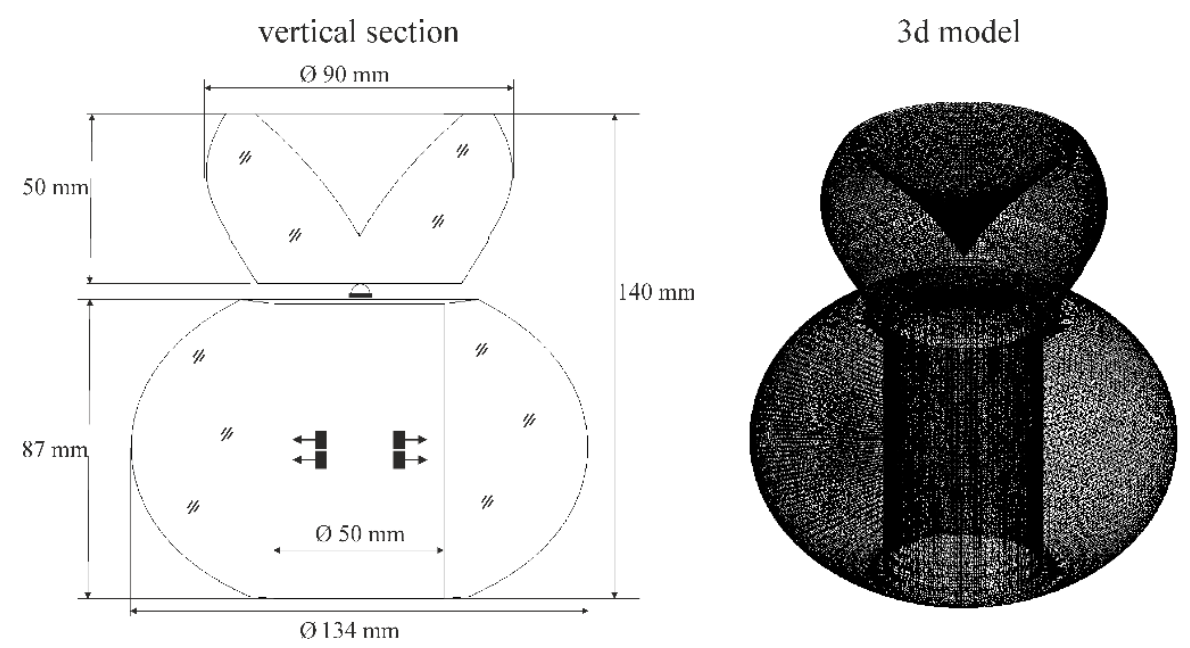

Fig. 11. The designed optical system.

A number of experiments were conducted in which the distance between Li-Fi systems in the horizontal plane varied from $3 \mathrm{~m}$ to $10 \mathrm{~m}$, and the height varied from $-0.8 \mathrm{~m}$ to $+0.8 \mathrm{~m}$. Figure 12 shows the dependence of the received signal power for a calculated optical system with a freeform surface and for a wide-angle spherical optical system at different positions in space. 


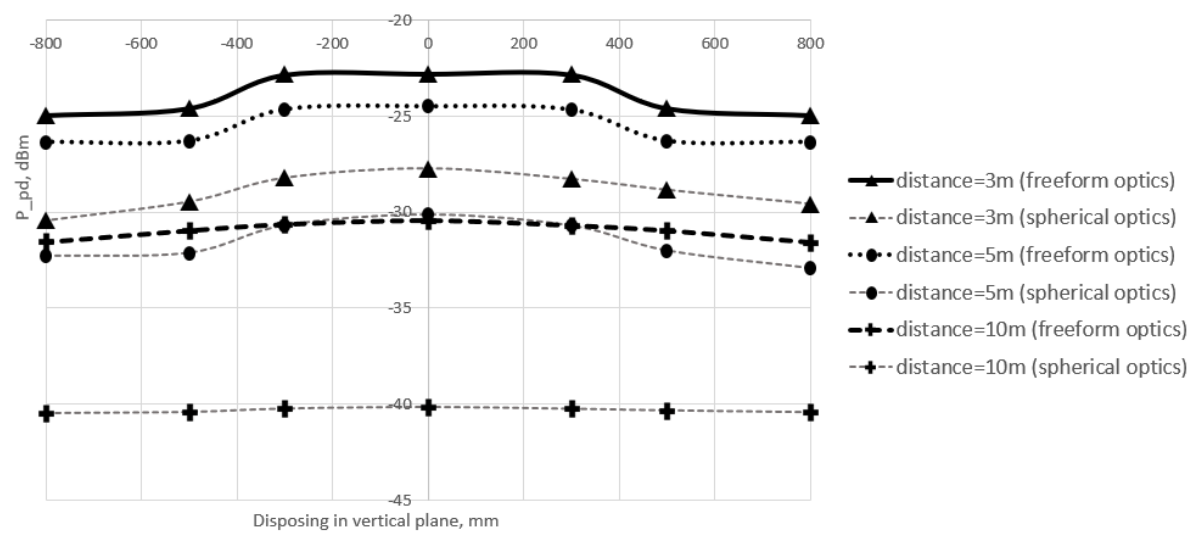

Fig. 12. The dependence of the received signal power for different positions

At a distance of $3 \mathrm{~m}$ between the systems, when using optical systems with freeform surfaces, the received signal level is not lower than $-25 \mathrm{dBm}$. The use of spherical optics shows a decrease in the signal level to $-30 \mathrm{dBm}$ at the same spatial positions. When studying the signal level at a distance of $5 \mathrm{~m}$, the optical system with freeform surfaces also shows better results compared to spherical optics: $-27 \mathrm{dBm}$ and $-32 \mathrm{dBm}$, respectively. At a distance of $10 \mathrm{~m}$, spherical optics shows low performance and the signal level is $-40 \mathrm{dBm}$; on the other hand, the optical system with a freeform surface provides a signal level of at least $-32 \mathrm{dBm}$. Thus, the use of specially designed optical systems with freeform surfaces for both the receiver and the source shows good performance compared to the use of optical systems with spherical surfaces.

\section{Conclusion}

In this paper, we propose a solution that makes it possible to modify the mapping method of input and output rays for calculating the optical system for a $\mathrm{Li}$-Fi module by introducing an integrated model of a source. The source optical system is a refractive optical system with an internal cylindrical surface and an external freeform surface. The receiver optical system contains two freeform refractive surfaces. The developed optical systems can be grouped into a hybrid optical system and placed on top of each other, providing a convenient location of the radiation sources and the receiver inside. The work shows the comparison of the performance of the calculated systems with a spherical optical system. This calculation method can be applied to real optical systems used in the Li-Fi technology of communication between vehicles.

\section{References}

1. Valavanidis, A.: The Shift to Diesel Fuel Engines and How the Emission Scandal of Diesel Vehicles Unfolded. World Energy Consumption of Transportation Sector. Scientific Reviews. vol. 1. pp.1-26 (2018). 
The Application of Integral Source Model in The Design of Freeform Optics... 13

2. World Health Organization (WHO), https://www.who.int/violence_injury_prevention/road_safety_status/report/ru/, last accessed 24.07.2020.

3. Demba, A., Möller, D. P. F. Vehicle-to-vehicle communication technology. 2018 IEEE International Conference on Electro/Information Technology (EIT). - IEEE, - pp. 0459-0464 (2018).

4. NHTSA. Vehicle-to-Vehicle Communication. https://www.nhtsa.gov/technology-innovation/vehicle-vehicle-communication, last accessed 24.07.20

5. Birdsall M. Google and ITE: The road ahead for self-driving cars. Institute of Transportation Engineers. ITE Journal. T. 84. №. 5. p. 36. (2014).

6. Alam, M., Ferreira, J., Fonseca, J. Introduction to intelligent transportation systems. Intelligent Transportation Systems. - Springer, Cham. pp. 11-13. (2016).

7. IEEE 1609 - Family of Standards for Wireless Access in Vehicular Environments (WAVE), https://www.standards.its.dot.gov/Factsheets/Factsheet/80, last accessed 24.07.20

8. Jin, W. L. et al. SPIVC: A Smartphone-based inter-vehicle communication system. Proceedings of transportation research board annual meeting (2012).

9. Boukerche, A. et al. Vehicular ad hoc networks: A new challenge for localization-based systems. Computer communications. T. 31. №. 12. pp. 2838-2849. (2008)

10. Haas, H. LiFi is a paradigm-shifting 5G technology. Reviews in Physics. T. 3. pp. 26-31. (2018).

11. Haas, H. et al. What is lifi? Journal of lightwave technology. T. 34. №. 6. pp. 1533-1544. (2015).

12. Tsonev, D., Videv, S., Haas, H. Light fidelity (Li-Fi): towards all-optical networking. Broadband Access Communication Technologies VIII. International Society for Optics and Photonics. T. 9007. pp. 900702. (2014).

13. Islim, M. S., Haas, H. Modulation techniques for Li-Fi. ZTE communications. T. 14. №. 2. pp. 29-40. (2019)

14. Ferreira, R. X. G. et al. High bandwidth GaN-based micro-LEDs for multi-Gb/s visible light communications. IEEE Photonics Technology Letters. T. 28. №. 19. pp. 2023-2026. (2016).

15. Wu, X., Safari, M., Haas, H. Access point selection for hybrid Li-Fi and Wi-Fi networks. IEEE Transactions on Communications. T. 65. №. 12. pp. 5375-5385. (2017).

16. Nachimuthu, S., Pooranachandran, S., Aarthi, B. S. Design and implementation of a vehicle to vehicle communication system using Li-Fi technology. International Research Journal of Engineering and Technology (IRJET). T. 3. №. 05. (2016)

17. Shieh, W. Y. et al. Investigation of vehicle positioning by infrared signal-direction discrimination for short-range vehicle-to-vehicle communications. IEEE Transactions on Vehicular Technology. T. 67. №. 12. pp. 11563-11574. (2018).

18. Kulkarni, S., Darekar, A., Shirol, S. Proposed framework for V2V communication using LiFi technology. 2017 International Conference on Circuits, Controls, and Communications (CCUBE). IEEE. pp. 187-190. (2017).

19. Yogarayan, S. et al. A comprehensive study of vehicle communication framework in Malaysia. Journal of Physics: Conference Series. IOP Publishing. T. 1502. №. 1. p. 012012. (2020).

20. Yeasmin, N., Zaman, R., Mouri, I. J. Traffic control management and road safety using vehicle to vehicle data transmission based on Li-Fi technology. International Journal of Computer Science, Engineering and Information Technology. T. 6. №. 3/4. pp. 1-7. (2016).

21. Kirrbach, R., Jakob, B., Noack, A. Introducing Advanced Freeform Optic Design to Li-Fi Technology. PHOTOPTICS. pp. 248-254. (2019). 
14 N. Bogdanov, I. Potemin et.al

22. Kirrbach, R., Faulwaßer, M., Jakob, B. Non-rotationally Symmetric Freeform FresnelLenses for Arbitrary Shaped Li-Fi Communication Channels. 2019 Global LIFI Congress (GLC). IEEE. pp. 1-6. (2019).

23. Wang, K. et al. New reversing design method for LED uniform illumination. Optics Express. Vol. 19, N. 104. pp. A830-A840. (2011).

24. Ding, Y. et al. Freeform LED lens for uniform illumination. Optics Express. Vol. 16, N 17. pp. 12958-12966. (2008).

25. Kirrbach, R. et al. Monolitic Hybrid Transmitter-Receiver Lens for Rotary On-Axis Communications. Applied Sciences. T. 10. №. 4. p. 1540. (2020)

26. G. S. Landsberg: Optics, Nauka publishing house, Moscow, (1976).

27. Bogdanov, N. N., Zhdanov, D. D., Potemin, I. S. Method of calculating the optical element for a low-intensity obstruction light. Izv.vuzov. Priborostoenie. Vol. 63, No. 7. pp. 157-166. (2020).

28. Hybrid Light Simulation Software Lumicept: https://integra.jp/en/products/lumicept, last accessed 24.07.2020.

29. Osram Opto Semiconductors, https://www.osram.com/ecat/OSLON®\%20Piccolo\%20SFH\%204170S\%20A01/com/en/cl ass_pim_web_catalog_103489/global/prd_pim_device_12764899/

30. Hamamatsu, https://www.hamamatsu.com/eu/en/product/type/S10784/index.html ,last accessed 24.07.2020

31. Solid Works, https://www.solidworks.com/ru, last accessed 24.07.2020. 